\title{
Crescimento Pós-Traumático após Divórcio: Contribuição dos Valores para Além das Variáveis Demográficas
}

\author{
Ricardo Neves Couto ${ }^{1, *}$ (D), Patrícia Nunes da Fonseca ${ }^{2}$ (D, Valeschka Martins Guerra ${ }^{3}$ (D), \\ \& Valdiney Veloso Gouveia ${ }^{2} \bullet$ \\ ${ }^{1}$ Universidade Federal do Delta do Parnaiba, Parnaiba, PI, Brasil \\ ${ }^{2}$ Universidade Federal da Paraíba, João Pessoa, PB, Brasil \\ ${ }^{3}$ Universidade Federal do Espirito Santo, Vitória, ES, Brasil
}

\begin{abstract}
RESUMO - Objetivou-se verificar em que medida os valores humanos explicam o crescimento pós-traumático no contexto do divórcio, controlando o efeito das variáveis idade, sexo e tempo de divorciado. Responderam ao Inventário de Crescimento Pós-Traumático, Questionário de Valores Básicos e questões demográficas 200 pessoas que vivenciaram o divórcio $\left(M_{\text {idade }}=38,4 ; \mathrm{DP}=11,51\right)$. Entre as variáveis demográficas, unicamente o sexo foi importante para explicar o crescimento, mais evidente entre mulheres. Ademais, os valores humanos se mostraram um construto importante. Subfunções sociais (interativa e normativa) e a de existência foram as mais importantes; entre os valores pessoais, unicamente os da subfunção realização lograram fazê-lo. Concluindo, estes achados foram discutidos à luz da literatura, sinalizando a importância dos valores humanos.
\end{abstract}

PALAVRAS-CHAVE: crescimento pós-traumático, valores humanos, divórcio

\section{Posttraumatic Growth After Divorce: Contribution of Values Beyond Demographic Variables}

\begin{abstract}
This study investigated to which extent human values explain posttraumatic growth in the context of divorce, even controlling by age, gender and divorce time. Two-hundred people who experienced the divorce responded to Posttraumatic Growth Inventory, Basic Values Questionnaire and demographic questions $\left(M_{\text {age }}=38.4, S D=11.51\right)$. Among the demographic variables only the gender was important to explain the growth, more evident among women. In addition, human values proved to be an important construct to explain. Social (interactive and normative) and existence subfunctions were the most important; between personal values, only those of the promotion subfunction did so. In conclusion, these findings were discussed at light of the literature, pointing out the importance of human values.
\end{abstract}

KEYWORDS: posttraumatic growth, human values, divorce

A dissolução do casamento tem crescido no Brasil, atingindo o número recorde em 2018. Os dados divulgados em 2019 acerca das Estatísticas do Registro Civil pelo Instituto Brasileiro de Geografia e Estatística (IBGE, 2019) contabilizam 385.146 divórcios. Neste cenário, constata-se que os cônjuges têm encontrado no divórcio a saída para um casamento conflituoso (Figueiredo \& Figueiredo, 2014), embora existam diversas estratégias de resolução de conflitos e manutenção do relacionamento conjugal, a exemplo da terapia de casal e a mediação familiar. Contudo, a decisão de se divorciar não é fácil, requerendo do indivíduo um posicionamento pessoal frente a diversas demandas (e.g., aceitação da divisão de bens, disposição para continuar um relacionamento coparental, reorganização financeira, regulação emocional de forma a lidar com as emoções de mágoa, raiva ou culpa) e uma adaptação à nova realidade.

O divórcio vem sendo apontado como um dos eventos mais estressores da vida adulta (Holmes \& Rahe, 1967),

*E-mail: r.nevescouto@gmail.com

- Submetido: 01/05/2018; Aceito: 08/09/2020. 
considerado como potencialmente traumático (YárnozYaben et al., 2015). Adicionalmente, pode apresentar efeitos adversos no bem-estar psicológico dos ex-cônjuges (Lamela et al., 2016) e no ajustamento psicológico dos filhos (Averdijk et al., 2012). Ademais, na tarefa de redescobrir o que significa a desintegração do casal, os indivíduos podem continuar convivendo com sentimentos negativos (e.g., raiva, culpa), além de frustações por ter sonhos e planos interrompidos (Alves et al., 2014). Ness e caso, o desafio será ressignificar o evento, buscando significados positivos para o divórcio, integrando-os em uma narrativa coerente sem sentimentos de ressentimento direcionados ao ex-parceiro, possibilitando, assim, que o término maximize a canalização das energias para novos projetos (Yárnoz-Yaben et al., 2015).

Faz-se necessário conhecer como os indivíduos lidam com essa busca de novas possibilidades e aprendizagens, aumentando a probabilidade de vivenciar mudanças positivas após situações adversas. $\mathrm{O}$ fenômeno, conceituado como crescimento pós-traumático (CPT), conduz as pessoas à encararem as dificuldades como oportunidades para a perseverança, desenvolvimento de novas aprendizagens e competências, além de crescer por meio do sofrimento, assumindo uma nova e saudável perspectiva de vida (Lau et al., 2013; Subandi et al, 2014; Waugh et al., 2018).

Ressalta-se que o CPT já foi estudado em amostra com pessoas divorciadas, abordando especificamente o divórcio como um gatilho para novas rotas de desenvolvimentos e descobertas (Lamela et al., 2014). A partir disso, asseverase a importância de seguir realizando pesquisas no âmbito do divórcio a fim de identificar variáveis que possam ajudar a compreender o caráter adaptativo e construtivo, essencial ao processo de reorganização dessa experiência possivelmente traumática. Assim, estima-se que os valores humanos, construto central nos estudos da Psicologia Social (Estramiana et al, 2013), e diferenças demográficas de pessoas que vivenciaram o processo de divórcio possam explicar o CPT no âmbito do divórcio. A propósito, os valores têm contribuído na compreensão de diversos comportamentos, decisões e avaliações apresentadas pelas pessoas (Gouveia, 2013; Rokeach, 1973), tendo natureza benevolente que evidencia os aspectos positivos do ser humano (Godoy \& Oliveira-Monteiro, 2015).

Dado o exposto, levantam-se as seguintes questões: poderiam o sexo, a idade e o tempo de divórcio influenciar no CPT? Quais são os valores humanos endossados por pessoas que vivenciam mudanças positivas no âmbito do divórcio? A fim de responder a essas perguntas e proporcionar contribuições acerca de intervenções positivas no contexto do divórcio, o presente estudo objetivou verificar em que medida os valores humanos explicam o crescimento póstraumático no contexto do divórcio, controlando o efeito das variáveis idade, sexo e tempo de divorciado. Neste marco, oferecem-se inicialmente conceituações dos construtos abordados.

\section{Crescimento Pós-Traumático}

O crescimento pós-traumático (CPT) é definido por Triplett et al. (2012) como "uma consequência potencial do esforço cognitivo de redefinição de crenças pessoais e de reconstrução da sua noção de mundo" (p. 400), vivenciada após uma experiência considerada traumática pelo indivíduo. Essa reavaliação pode gerar mudanças que contribuam para que o indivíduo atribua sentido ao seu sofrimento e um aumento de satisfação com a vida (Bluvtein et al., 2012; Lau et al., 2013; Madeira, 2013; Tedeschi et al., 1998). Portanto, quanto mais o indivíduo percebe que o evento traumático desafia suas crenças centrais a ponto de sentir necessidade de reavaliá-las, maior a chance de um crescimento póstraumático (Triplett et al., 2012).

Tedeschi e Calhoun (1996) propuseram uma estrutura teórica do CPT com base em três dimensões: (1) mudança de significado do self ou autopercepção - melhor percepção de suas forças e mais confiança para seguir a vida, mesmo com futuras adversidades; (2) mudança de significado das relações com os outros - abertura para aproximações e apoio social, fortalecimento e seleção das amizades; e (3) mudança na filosofia de vida - mudanças nas prioridades em diferentes domínios da vida, valorização da própria vida, pequenas coisas e momentos, envolvimento com questões espirituais e existenciais.

Empiricamente, pesquisas fundamentadas sobretudo na perspectiva da Psicologia Positiva, com o uso do Posttraumatic Growth Inventory (ver adaptação desta medida para o Brasil em Medeiros et al., 2017; Taku et al., 2012; Ullman, 2014), demonstram que o CPT é um construto multidimensional, que agrupa cinco dimensões: (1) aumento da apreciação de vida no geral, (2) relações interpessoais mais significativas, (3) aumento do sentimento de força pessoal, (4) mudanças de prioridades e (5) uma vida espiritual e existencial mais rica. Considera-se também um fator geral do CPT, que reúne todos os itens dessa medida (Groleau et al., 2013; Laaser et al., 2017; Zhang et al., 2013).

As experiências que caracterizam o CPT são dependentes do nível individual de autoconhecimento, no que diz respeito ao reconhecimento das próprias forças e da ressignificação que o indivíduo faz da vivência estressora. Observa-se também a importância do processo de ruminação, tanto deliberada (com a contemplação do acontecimento) quanto imediata após o trauma, ambas com o objetivo de minimizar a angústia emocional (Ramos \& Leal, 2013; Zhang et al., 2013). Além da ruminação, o sentimento de gratidão pelas oportunidades dadas, mesmo em situações adversas, potencializa as formações de cognições positivas que ajudam a restabelecer o significado da vida (Wu et al., 2014; Zhou \& Wu, 2015). Entretanto, dois conjuntos de variáveis podem ser também importantes para compreender a manifestação desse crescimento, como a seguir são tratadas. 


\section{Características Demográficas e o Crescimento Pós-Traumático}

Quanto às caraterísticas demográficas, estudos apontam que, de maneira geral, mulheres vivenciam maiores níveis de CPT do que os homens (Ramos \& Leal, 2013; Tedeschi $\&$ Calhoun, 2004). Elas buscam mais ajuda e se sentem mais confortáveis para compartilhar as experiências com outras pessoas, sobretudo com aquelas que passaram por situações semelhantes, enquanto os homens optam por não compartilhar sentimento de tristeza, com receio de demonstrar fracasso.

No que se refere à idade e ao tempo de divórcio, observase que a necessidade de ressignificação após o trauma faz com que as pessoas mais novas apresentem maiores níveis de crescimento. Isso é teoricamente justificado, uma vez que as crenças sobre o mundo e sobre si ainda são maleáveis, passivas de modificações e ressignificações até a sua consolidação (Fonseca, 2011; Vázquez et al., 2013). Especificamente no âmbito do divórcio, com o passar do tempo, os ex-parceiros buscam seguir sua vida e diminuem as consequências negativas do divórcio. Focalizam suas energias em novos projetos pessoais e minimizam os sentimentos negativos frente ao ex-parceiro (Yárnoz-Yaben et al., 2015), fazendo com que a adaptação se torne menos dolorosa com o tempo. Além disso, novas estratégias e decisões passam a ser consideradas no cotidiano, o que podem ocasionar maiores níveis de bem-estar psicológico (Lamela et al., 2016).

Uma variável demográfica que tem sido discutida, mas sem resultados consistentes, diz respeito à dimensão mais espiritual. Estima-se que esta, por vezes definida como espiritualidade e conhecimentos religiosos, possa auxiliar na explicação do CPT (Subandi et al., 2014). $\mathrm{N}$ a mesma direção, conjetura-se a relevância do apoio recebido por grupos de pertença, a exemplo de familiares e amigos, principalmente os novos, que potencializam uma narrativa nova e integradora da vida (Duarte, 2012). Como anteriormente se comentou, os achados a respeito não são conclusivos, principalmente no âmbito do divórcio (Lindstrom et al., 2013), o que motiva a considerar uma variável que pode integrar de forma mais sistemática essas orientações, isto é, os valores humanos. Estes serão tratados a seguir.

\section{Valores Humanos e Crescimento Pós-Traumático}

Inicialmente, há que se dizer que é considerada nesta oportunidade como embasamento para os valores a Teoria Funcionalista dos Valores Humanos (TFVH), que tem demonstrado um potencial explicativo nos contextos nacional e internacional (Gouveia, 2016). El a foi proposta por Gouveia $(1998,2003,2013)$, compreendendo uma teoria integradora sobre os valores humanos, além de ser parcimoniosa e fundamentada na concepção das funções principais dos valores. Suas evidências de adequação abrangem não apenas a realidade brasileira, com aproximadamente 50 mil pessoas, mas inclui mais de 50 países dos cinco continentes (Medeiros, 2011; Soares, 2015).

O principal foco dessa teoria são valores como aspectos psicológicos, que cumprem duas funções: (1) guiar os comportamentos, representada pela forma como o indivíduo orienta seu comportamento, cognição e afeto, podendo ser social (com metas sociais representantes do indivíduo na sociedade), central (orientando a busca do propósito geral da vida) e pessoal (indivíduo busca metas por si mesmo); e (2) representar cognitivamente as necessidades humanas, definida pelo tipo de motivação do indivíduo, podendo ser materialista (com ideias práticas e imediatas) e humanitária (comportamentos são dirigidos a metas gerais e inovadoras; Gouveia et al., 2014). O cruzamento $d$ as funções origina seis subfunções (experimentação, realização, existência, suprapessoal, interativa e normativa), resumidas a seguir, segundo Gouveia (2013).

A subfunção experimentação (orientação pessoal e motivador idealista) representa a necessidade fisiológica de satisfação, em sentido amplo, ou o princípio do prazer (hedonismo). Contribui para a promoção de mudança e inovação na estrutura das organizações sociais. Os valores que a representam são emoção, prazer e sexualidade.

As pessoas orientadas pela subfunção realização (orientação pessoal, motivador materialista) focam em realizações materiais, pessoais e imediatas, voltadas para o aqui e agora. É constituída pelos valores específicos êxito, poder e prestígio.

A subfunção existência (orientação central e motivador materialista) representa cognitivamente as necessidades fisiológicas mais básicas. Seu propósito principal é assegurar as condições essenciais para a sobrevivência biológica e psicológica do indivíduo. Os valores que a constituem são estabilidade social, saúde e sobrevivência.

A subfunção suprapessoal (orientação central e motivador humanitário) representa as necessidades de autorrealização, cognição e estética. Seus valores específicos indicam a importância de ideias abstratas, com menor ênfase em coisas concretas e materiais. Fazem parte desta subfunção os valores beleza, conhecimento e maturidade.

A subfunção interativa representa as necessidades de pertença, amor e afiliação, promovendo o estabelecimento e a manutenção das relações interpessoais por parte do indivíduo, que é orientado a relações íntimas estáveis. Os valores desta subfunção são afetividade, apoio social e convivência.

A subfunção normativa (orientação social e motivador materialista) representa a necessidade de controle e as pré-condições imprescindíveis para alcançar todas as necessidades. Concebe a ideia de preservação da cultura e das normas convencionais, sendo mais priorizada 
por pessoas mais velhas e/ou criadas em contextos convencionais. É constituída pelos valores obediência, religiosidade e tradição.

Encontram-se na literatura diversas evidências empíricas da contribuição dos valores humanos na perspectiva da TFVH (Gouveia, 2016). As subfunções valorativas já foram utilizadas, por exemplo, com o fim de explicar a satisfação conjugal (Almeida, 2016), a satisfação com a vida em jovens (Marques et al., 2017) e o perdão conjugal (Fonsêca et al., 2017). No que diz respeito ao CPT, Medeiros et al. (2016) demonstraram o poder explicativo das subfunções valorativas em pessoas da população geral que passaram por diferentes traumas na vida e Couto (2019) verificou a explicação dos valores sociais, mediados por forças de caráter, no CPT em uma amostra de pessoas que passaram por luto. Contudo, nada foi encontrado quando ao contexto específico do divórcio, reforçando a importância do presente estudo.

Em suma, os valores humanos são princípios-guia desejáveis que têm o papel de assegurar a sobrevivência, manutenção e evolução da sociedade, explicando atitudes e comportamentos de seus membros (Gouveia, 2019). No entanto, não foram encontradas evidências específicas de mudanças positivas pós-divórcio, logo, dada a magnitude da problemática apresentada, julga-se pertinente a realização da pesquisa e apresentação, a seguir, de dados empíricos, uma vez que tais informações podem contribuir para a melhor compreensão de como os valores podem ser estimulados para favorecer habilidades de superação e adaptação positiva frente ao divórcio.

\section{MÉTODO}

\section{Participantes}

Contou-se com uma amostra de conveniência (não probabilista), considerando-se como critérios de inclusão ser maior de 18 anos e ter passado por um processo de divórcio. Participaram 200 indivíduos divorciados, oriundos de estados da região Nordeste: Paraíba (66), Piauí (65), Ceará (39), Maranhão (22), Pernambuco (18), Rio Grande do Norte (6) e Bahia (4), distribuídos equitativamente entre os sexos, com idades entre 19 e 71 anos $(\mathrm{M}=38,4, \mathrm{DP}=$ 11,51), o tempo médio de divórcio foi de 5,8 anos ( $\mathrm{DP}=$ 5,01; amplitude de 1 ano a 33 anos). Destes, 31\% tinham curso de pós-graduação e $25,5 \%$ informaram renda familiar de $\mathrm{R} \$ 1.801,00$ a $\mathrm{R} \$ 2.700,00$.

\section{Instrumentos}

\section{Inventário de Crescimento Pós-Traumático (ICP)}

Desenvolvido por Tedeschi e Calhoun (1996), o ICP foi adaptado para o Brasil por Medeiros et al. (2017). Avalia o grau de mudanças positivas percebidas pelo indivíduo após um acontecimento adverso e é constituído por 21 itens, distribuídos em 5 fatores: Relação com os outros (Itens 6,8,9,15,16,20 e 21), Novas possibilidades (Itens 3,7,11,14 e 17), Mudança Pessoal (Itens 4,10,12 e 19), Mudança Espiritual (Itens 5 e 18) e Apreciação da Vida (Itens 1,2 e 13). Seu Fator Geral, somatório de todos os itens, corresponde ao índice de crescimento pós-traumático. Os itens são respondidos em escala de 6 pontos, variando de 0 (Não experimentei mudança como resultado desse trauma) a 5 (Mudei completamente), expressando em que medida a pessoa considera ter mudado em decorrência de determinado trauma (e.g., "Dou mais valor à minha vida"; "Descobri que sou mais forte do que pensava"). As dimensões do ICP apresentaram alfa de Cronbach entre 0,70 (Apreciação da vida) e 0,84 (Relação com os outros), além de 0,92 para o Fator Geral.

\section{Questionário dos Valores Básicos (QVB)}

Elaborado por Gouveia (1998, 2003, 2013), esse instrumento é composto por 18 itens ou valores específicos (Gouveia et al., 2014). Para cada um dos valores são apresentados dois descritores (e.g., Convivência. Conviver diariamente com os vizinhos; fazer parte de algum grupo, como social e esportivo). Esses valores são distribuídos equitativamente entre as seis subfunções, sendo avaliados pelo respondente em razão de sua importância como um princípio-guia em sua vida, utilizando uma escala de 7 pontos, variando de 1 (Totalmente não importante) a 7 (Extremamente importante). Suas qualidades psicométricas têm sido satisfatórias para fins de pesquisas em contextos brasileiros e internacionais (Medeiros, 2011; Soares, 2015). Por exemplo, seus alfas de Cronbach costumam estar entre 0,50 e 0,70, coeficientes compatíveis com outras medidas de valores (e.g., Schwartz, 2005).

\section{Questionário Demográfico}

Incluíram-se perguntas com o fim de caracterizar os participantes do estudo, além de avaliar em que medida poderiam contribuir para explicar as pontuações do CPT no âmbito do divórcio. Especificamente, perguntou-se sobre idade, escolaridade, renda, sexo e tempo de divorciado.

\section{Procedimento}

Os instrumentos foram aplicados em locais públicos (e.g. praças, shoppings) e online na plataforma Google Docs, tendo -se em conta que tais formas de coletas têm apresentado resultados similares (Brock et al., 2012). Os 
participantes que concordaram em colaborar atestaram o seu consentimento por meio do Termo de Consentimento Livre e Esclarecido (TCLE), sendo informados acerca do objetivo geral do estudo, além do caráter anônimo e voluntário da pesquisa, podendo desistir a qualquer momento sem sofrer ônus. Em média, 15 minutos foram suficientes para concluir a participação.

\section{Análise de Dados}

Com o SPSS (versão 23), foram realizadas estatísticas descritivas (média, desvio-padrão e estimativas de frequências) para caracterização dos participantes, além de correlações $r$ de Pearson, a fim de identificar a relação dos valores humanos com o CPT no âmbito do divórcio.
Posteriormente, também foram calculadas regressões múltiplas hierárquicas que permitiram identificar o poder preditivo das variáveis demográficas e subfunções valorativas na explicação do CPT em pessoas que passaram por divórcio.

\section{Considerações Éticas}

O projeto que abarca esse estudo teve a aprovação do Comitê de Ética de uma universidade pública da Paraíba (CAAE: 46788315.1.0000.5188; Protocolo $\mathrm{n}^{\circ}$ 0405/15). Procurou-se seguir as recomendações éticas relacionadas às pesquisas com seres humanos, conforme o disposto nas Resoluções 466/2012 e 510/16 do Conselho Nacional de Saúde.

\section{RESULTADOS}

Com o propósito de conhecer como as seis subfunções valorativas e as dimensões do CPT se correlacionam, calcularam-se coeficientes de correlação $r$ de Pearson. Os resultados são descritos na Tabela 1 , onde se observa que o fator geral do CPT se correlacionou positivamente com todas as subfunções valorativas $(p<0,001)$, variando de 0,16 (experimentação) a 0,37 (existência). Conforme a tabela, resultados similares foram observados para todas as dimensões do CPT, isto é, elas apresentaram correlações diretas com todas as subfunções de valores, sendo as únicas exceções as dimensões do crescimento pós-traumático relações com os outros e mudanças pessoais, que não apresentaram correlações com a subfunção experimentação.

Considerando o padrão de correlação entre valores e fatores de crescimento pós-traumático, decidiu-se avaliar em que medida os valores podem explicar ess e crescimento, controlando o efeito das variáveis demográficas e realizando análises de regressão múltipla hierárquica. Nesta direção, primeiramente foram inseridas no modelo as variáveis demográficas previamente analisadas (i.e., idade, sexo e tempo divorciado), introduzindo no segundo passo as subfunções valorativas que se correlacionaram com as pontuações nas dimensões de crescimento pós-traumático. Os resultados d a análise são mostrados na Tabela 2 .

Quando as variáveis demográficas (idade, sexo e tempo divorciado) foram inseridas no modelo, observaramse resultados significativos apenas para a dimensão mudanças pessoais $\left[F(3 ; 196)=2,67, p=0,04, R^{2}=0,04\right]$. Entretanto, quando os valores humanos foram inseridos no segundo passo, isto é, controlando o efeito das variáveis

Tabela 1

Estatísticas descritivas e correlações entre as subfunções valorativas e as dimensões do crescimento pós-traumático (CPT).

\begin{tabular}{|c|c|c|c|c|c|c|c|c|c|c|c|c|c|c|}
\hline Dimensões & $\mathbf{M}$ & DP & 1 & 2 & 3 & 4 & 5 & 6 & 7 & 8 & 9 & 10 & 11 & 12 \\
\hline 1. CPT & 71,59 & 19,44 & 0,92 & & & & & & & & & & & \\
\hline 2. REL & 21,60 & 7,95 & $0,88^{* *}$ & 0,84 & & & & & & & & & & \\
\hline 3. NP & 18,01 & 4,64 & $0,85 * *$ & $0,63 * *$ & 0,72 & & & & & & & & & \\
\hline 4. MP & 14,96 & 4,40 & $0,83 * *$ & $0,58 * *$ & $0,70 * *$ & 0,80 & & & & & & & & \\
\hline 5. ME & 6,23 & 3,11 & $0,72 * *$ & $0,61 * *$ & $0,49 * *$ & $0,54 * *$ & 0,79 & & & & & & & \\
\hline 6. AV & 10,78 & 3,30 & $0,78 * *$ & $0,54 * *$ & $0,70 * *$ & $0,67 * *$ & $0,42 * *$ & 0,70 & & & & & & \\
\hline 7. Inter & 16,83 & 2,81 & $0,33 * *$ & $0,37 * *$ & $0,20^{*}$ & $0,24 * *$ & $0,26^{* *}$ & $0,18^{*}$ & 0,61 & & & & & \\
\hline 8. Norm & 16,20 & 3,50 & $0,36 * *$ & $0,34 * *$ & $0,21 *$ & $0,23 *$ & $0,43 * *$ & $0,31 * *$ & $0,43 * *$ & 0,69 & & & & \\
\hline 9. Supr & 16,59 & 2,40 & $0,24 * *$ & $0,15^{*}$ & $0,20^{*}$ & $0,26 * *$ & $0,12 *$ & $0,29 * *$ & $0,38 * *$ & $0,34 * *$ & 0,46 & & & \\
\hline 10. Exist & 18,01 & 2,76 & $0,37 * *$ & $0,28 * *$ & $0,31 * *$ & $0,40 * *$ & $0,18 * *$ & $0,36 * *$ & $0,47 * *$ & $0,33 * *$ & $0,47 * *$ & 0,63 & & \\
\hline 11. Exp & 14,57 & 3,28 & $0,16^{*}$ & 0,12 & $0,20^{*}$ & $0,16^{*}$ & 0,01 & $0,17 * *$ & $0,18^{* *}$ & $-0,05$ & $0,30 * *$ & $0,31 * *$ & 0,55 & \\
\hline 12. Real & 13,78 & 3,37 & $0,29 * *$ & $0,24 * *$ & $0,26 * *$ & $0,30 * *$ & $0,14 *$ & $0,24 * *$ & $0,28 * *$ & $0,19 *$ & $0,33 * *$ & $0,33 * *$ & $0,48 * *$ & 0,59 \\
\hline
\end{tabular}

Nota. ${ }^{* *} p<0,001 ;{ }^{*} p<0,05$ (teste unicaudal); $\mathbf{M}=$ Média; $\mathbf{D P}=$ Desvio padrão; $\mathbf{1}=$ Fator geral do CPT; $\mathbf{2}=$ Relação com outros; $\mathbf{3}=$ Novas Possibilidades; 4 = Mudanças Pessoais; 5 = Mudanças Espirituais; 6 = Apreciação da vida; 7 = Subfunção Interativa; 8 = Subfunção Normativa; $9=$ Subfunção Suprapessoal; 10 = Subfunção Existência; 11 = Subfunção Experimentação; 12 = Subfunção Realização. Os valores nas diagonais correspondem aos coeficientes de consistência interna mensurados através do alfa de Cronbach. 
Tabela 2

Análise de regressão hierárquica dos preditores das dimensões do crescimento pós-traumático

\begin{tabular}{|c|c|c|c|c|c|c|}
\hline Variáveis & REL & NP & MP & ME & AV & CPT \\
\hline Passo 1 & $\beta$ & $\beta$ & $\beta$ & $\beta$ & $\beta$ & $\beta$ \\
\hline Idade & $-0,07$ & $-0,07$ & $-0,06$ & 0,05 & $-0,01$ & $-0,07$ \\
\hline $\operatorname{Sexo}^{\mathrm{a}}$ & 0,09 & 0,12 & $0,17 *$ & 0,13 & $0,14 *$ & $0,15^{*}$ \\
\hline Tempo divorciado & 0,18 & 0,07 & 0,14 & 0,10 & 0,08 & 0,17 \\
\hline$R^{2}$ & 0,03 & 0,02 & $0,04 *$ & 0,03 & 0,03 & 0,04 \\
\hline Passo 2 & $\beta$ & B & $\beta$ & $\beta$ & $\beta$ & $\beta$ \\
\hline Idade & $-0,05$ & $-0,07$ & $-0,08$ & 0,03 & $-0,05$ & $-0,07$ \\
\hline $\operatorname{Sexo}^{\mathrm{a}}$ & 0,09 & 0,10 & $0,15^{*}$ & 0,12 & 0,11 & $0,14 *$ \\
\hline Tempo divorciado & 0,07 & 0,03 & 0,10 & $-0,02$ & 0,01 & 0,06 \\
\hline Experimentação & - & 0,07 & $-0,06$ & - & 0,03 & 0,01 \\
\hline Realização & $0,15^{*}$ & 0,14 & $0,21^{*}$ & 0,07 & 0,11 & $0,17 *$ \\
\hline Existência & 0,10 & $0,20 *$ & $0,29 * *$ & $-0,03$ & $0,24 * *$ & $0,20 *$ \\
\hline Suprapessoal & $-0,09$ & $-0,02$ & 0,03 & - & 0,08 & $-0,04$ \\
\hline Interativa & $0,22 *$ & 0,01 & 0,01 & 0,08 & $-0,09$ & 0,09 \\
\hline Normativa & $0,21 *$ & 0,13 & 0,07 & 0,40 ** & $0,23 *$ & $0,24 *$ \\
\hline$R^{2}$ & $0,22 * *$ & $0,15^{* *}$ & $0,23 * *$ & $0,21 * *$ & $0,21^{* *}$ & $0,25^{* *}$ \\
\hline$\Delta R^{2}$ & 0,19 & 0,13 & 0,19 & 0,18 & 0,18 & 0,21 \\
\hline
\end{tabular}

Nota. ${ }^{* *} p<0,001 ; * p<0,05 ;{ }^{a}$ Masculino $=1$, Feminino $=2$. REL = Relações com os outros; $\mathrm{NP}=$ Novas possibilidades; $\mathrm{MP}=$ Mudanças pessoais; $\mathrm{ME}=$ Mudanças espirituais; $\mathrm{AV}=$ Apreciação da vida; $\mathrm{CPT}=$ Fator geral do Crescimento Pós-traumático.

demográficas, foi possível verificar que todos fatores do CPT foram explicados: relação com os outros $[F(8 ; 191)$ $\left.=6,44 ; p<0,001 ; R^{2}=0,22\right]$, novas possibilidades $[F$ $\left.(9 ; 190)=3,73 ; p<0,001 ; R^{2}=0,15\right]$, mudanças pessoais $[F$ $\left.(9 ; 190)=6,21 ; p<0,001 ; R^{2}=0,23\right]$, mudança espiritual $\left[F(7 ; 192)=7,40 ; p<0,001 ; R^{2}=0,21\right]$ e apreciação da vida $\left[F(9 ; 190)=5,58 ; p<0,001 ; R^{2}=0,21\right]$, além do fator geral $\left[F(9 ; 190)=7,11 ; p<0,001 ; R^{2}=0,25\right]$. Portanto, verificou-se incremento do modelo explicativo do CPT quando consideradas as subfunções valorativas.

Quanto às variáveis preditivas no modelo final, ressaltase que a idade e o tempo de divórcio não demonstraram qualquer poder preditivo dos fatores do CPT. Entretanto, no caso da explicação do fator geral da CPT, as mulheres apresentaram pontuações maiores que os homens. Esse fator também foi explicado pelas subfunções normativa, existência e realização. $\mathrm{O}$ fator relação com os outros foi explicado pelas subfunções interativa, normativa e realização; o fator mudanças pessoais foi explicado pelas subfunções existência e realização, além do sexo dos participantes, com mulheres pontuando mais do que os homens. Os fatores mudanças espirituais e apreciação da vida foram explicados pela subfunção normativa, sendo este último fator também explicado por existência. Finalmente, o fator denominado de novas possibilidades foi explicado unicamente pela subfunção existência.

\section{DISCUSSÃO}

A presente pesquisa teve como objetivo verificar em que medida os valores humanos poderiam explicar fatores de crescimento pós-traumático no âmbito do divórcio, depois de controlada a influência de características demográficas (idade, sexo e tempo de divorciado). Portanto, considera-se que este objetivo tenha sido alcançado. Ness e sentido, tais resultados contribuem para a construção do conhecimento acerca do $\mathrm{CPT}$, sugerindo que determinados valores podem favorecer o desenvolvimento de habilidades que contribuem para a superação do trauma no contexto de divórcio, dando ênfase ao que faz a vida ter sentido (Pacico \& Bastianello, 2014; Vázquez, 2013), como expresso por meio do valor existência.
A propósito do anteriormente comentado, verificouse que a idade ou o tempo de divórcio não influenciam no crescimento pós-traumático. Embora tenha sido um resultado diferente d o que se esperava (Duarte, 2012), entende-se que em todas as faixas etárias o processo de adaptação ao divórcio pode acontecer de maneira positiva. Jovens e adultos maduros estão abertos a novas aprendizagens e encaram a situação adversa do divórcio como uma oportunidade de aprender coisas novas, saber lidar com situações e perceber que a vida é feita de escolhas (mesmo algumas não obtendo o resultado desejado) e que existem outras disponíveis (Tedeschi \& Calhoun, 2004). A 
propósito, espera-se que os mais velhos atinjam facilmente um nível de maturidade que os auxilia a ressignificar o evento, além de perceberem que há outras pessoas (e.g., filhos, netos, amigos) que ainda estão ao seu lado e serão seus companheiros, devendo a energia ser voltada para esses, $\mathrm{h}$ aja vista que, atualmente, os idosos se sentem vigorosos e dispostos a buscar experiências novas e realizações (Duarte, 2012; Ramos \& Leal, 2013).

Quanto ao sexo, observam-se maiores pontuações das mulheres do que dos homens nos fatores mudanças pessoais, apreciação da vida e fator geral. A literatura explica esse resultado a partir da maior abertura delas, sobretudo com pessoas que passaram por situações semelhantes, fazendo com que apresentem maior engajamento no processo de luta e mudanças positivas (Ramos \& Leal, 2013; Tedeschi $\&$ Calhoun, 2004). Contrariamente, os homens, após o divórcio, sentem-se inseguros e desconfortáveis para compartilhar suas experiências traumáticas, com receio de demonstrar fracasso (Linley \& Joseph, 2004).

O tempo de divórcio não influenciou nos índices de CPT. Segundo Feeney e Monin (2008), o reconhecimento e a atitude de se permitir continuar vivendo facilita que as pessoas focalizem suas energias em novas oportunidades, buscando assim comportamentos, pensamentos e vivências que aumentem a autoestima. Cada pessoa passará por um processo, no qual após se reconhecer como solteira de novo, procura canalizar as suas energias para novos projetos, abandonando de vez pretensões de retaliação, vingança, ódio e ressentimento contra o ex-cônjuge (Menezes, 2009; Yárnoz-Yaben et al., 2015).

No tocante aos valores, pareceu evidente o papel desse construto no crescimento pós-traumático no contexto do divórcio. Foram particularmente importantes os valores sociais (subfunções interativa e normativa) e de existência. A subfunção interativa predisse o fator relação com os outros, coerente com a ideia de que seus valores acentuam as relações interpessoais (Gouveia, 2013, 2019). É possível que o suporte social apreciado por pessoas que se pautam nesses valores promova o desenvolvimento de novas perspectivas, com base na reavaliação cognitiva associada ao ato de compartilhar com os demais suas experiências, possibilitando a criação de uma nova e integradora narrativa de vida (Duarte, 2012). Ademais, pode aumentar a sensação de compaixão por outros que passam por dificuldades semelhantes (Calhoun \& Tedeschi, 2012; Tedeschi \& Calhoun, 1996).

A subfunção normativa predisse as pontuações em mudanças espirituais, apreciação da vida, relação com os outros e fator geral do CPT. Medeiros et al. (2018) encontraram que esta subfunção foi a que apresentou maior correlação com o CPT em uma amostra brasileira reunindo pessoas que passaram por diversos eventos traumáticos. Ness a oportunidade, salienta-se que os indivíduos ao enfrentarem um divórcio têm mais probabilidade de se tornarem engajados cognitivamente com questões existenciais sobre a morte e o sentido de vida, buscando satisfazer suas necessidades e priorizando a harmonia social, além de ressaltarem a manutenção de padrões tradicionais cotidianos, encontrando mais sentido em existir (Calhoun \& Tedeschi, 2012; Duarte, 2012; Gouveia et al., 2014).

Os valores centrais (suprapessoal e existência) influenciaram diferentemente o crescimento pós-traumático. Por um lado, a subfunção suprapessoal não predisse qualquer dimensão do CPT. Talvez não o tenha feito por, em razão das vivências de sofrimento, haver um crescimento natural das pessoas, maior maturidade e visão ampla do mundo, coerente com este tipo de orientação axiológica (Gouveia, 2013) e com o que se espera a partir da reorganização da experiência (Lamela et al., 2014). Por outro lado, existência explicou três fatores específicos (novas possibilidades, mudanças pessoais e apreciação da vida) e o fator geral do CPT no divórcio. Após uma vivência adversa, o indivíduo prioriza as condições básicas para sua sobrevivência biológica e psicológica, sentindo a necessidade de reexaminar as crenças, buscando reconstruir sua narrativa de vida (Triplett et al., 2012), o que facilita seu crescimento. Além disso, segundo Resende et al. (2008), quando a experiência de um evento é traumática, como em algumas situações o divórcio, leva o indivíduo a engatar no processamento cognitivo e alcançar um propósito à própria existência.

No caso dos valores pessoais, os resultados indicaram que a subfunção experimentação não predisse o CPT. Pode-se supor que isso se deva à especificidade da amostra, formada mais por jovens, que compartilham a priorização desta subfunção (Gouveia, 2013). Não obstante, há que se pensar também que pessoas que apreciam estes valores não se prendem a relações ou estilos de vida mais convencionais; talvez seu crescimento não se dê exatamente em razão de um divórcio, que pode não ser um evento traumático para alguns deles. Contudo, este aspecto carece de comprovação. Caberá a estudos futuros procurarem explicações para o papel desses valores, avaliando se sua não importância pode ser dever ao evento em si (divórcio). Por outro lado, em se tratando da subfunção realização, esta predisse os fatores relação com os outros, mudanças pessoais e o fator geral. Estes achados, quiçá, endossem a necessidade de as pessoas que vivenciam esse trauma afirmarem sua autoestima, o que converge com o que observaram Kulik e Heine-Cohen (2011), Lamela et al. (2014) e Thomas e Ryan (2008). Ess es autores sugerem que pessoas divorciadas, ao procurarem superar o fracasso da relação, experimentam maior sensação de autonomia, competência e habilidades de enfrentamento. Então, elas se reconhecem como mais fortes e confiantes para enfrentar adversidades futuras; a desilusão com o casamento passa a ser encarada como uma oportunidade de experiência e aprendizado (Lindstrom et al., 2013; Ramos \& Leal, 2013).

Apesar de os resultados consistentes deste estudo, corroborando em sua maioria o que tem sido observado na literatura, faz-se necessário contemplar suas limitações potenciais. Certamente, o viés da amostra, que foi de conveniência, limita a generalização dos achados para além de seu escopo (Cozby, 2003). O delineamento correlacional da pesquisa também não possibilita estabelecer relações de 
causa e efeito (Breakwell et al., 2010), apenas favorecendo conhecer que não é descabido pensar nos valores como um construto explicativo do crescimento pós-traumático. Por fim, poder-se-ia indagar acerca das magnitudes baixas das correlações, porém vale lembrar que o padrão observado é coerente com o que tem sido relatado em estudos da Psicologia (Hemphill, 2003).

Por fim, tomando o antes comentado, demandam-se pesquisas adicionais acerca desta temática, contemplando os valores, além de incluir outros construtos que podem potencialmente explicar o crescimento pós-traumático, como podem ser estilos parentais, traços de personalidade, autoestima e forças de caráter. Contudo, recomenda-se que sejam consideradas amostras maiores e mais diversas, incluindo pessoas de diferentes regiões do Brasil. Poderá ser ainda interessante considerar variáveis consequentes do crescimento pós-traumático, como podem ser adaptação ao divórcio, bem-estar subjetivo e esperança.

\section{REFERÊNCIAS}

Almeida, A. C. (2016). Satisfação Conjugal e Valores Humanos dos Casais de Famílias Intactas e Recasadas [Dissertação de mestrado, Universidade Federal da Paraíba]. Repositório Institucional da UFPB. https://repositorio.ufpb.br/jspui/handle/ tede $/ 8534$ ? locale $=$ pt_BR

Alves, A. P., Cúnico, S. D., Arpini, D. M., Smaniotto, A. C., \& Bopp, M. E. T. (2014). Mediação Familiar: Possibilitando Diálogos Acerca da Guarda Compartilhada. Pesquisas e Práticas Psicossociais, 9(2), 193-200.

Averdijk, M., Malti T., Eisner, M., \& Ribeaud, D. (2012). Parental Separation and Child Aggressive and Internalizing Behavior: An Event History Calendar Analysis. Child Psychiatry Human Development, 43(2), 184-200. https://doi.org/10.1007/s10578011-0259-9

Bardi, A., \& Schwartz, S. H. (2003). Values and Behavior: Strength and Structure of Relations. Personality and Social Psychology Bulletin, 29(10), 1207-1220. https://doi. org/10.1177/0146167203254602

Bluvtein, I., Moravchick, L., Sheps, D., Schreiber S., \& Bloch, M. (2012). Posttraumatic Growth, Posttraumatic Stress Symptoms and Mental Health among Coronary Heart Disease Survivors. Journal of Clinical Psychology in Medical Settings, 20(2), 164-172. https://doi.org/10.1007/s10880-012-9318-z.

Breakwell, G. M., Hammond, S., Fife-Schaw, C., \& Smith, J. A. (Orgs.). (2010). Métodos de pesquisa em psicologia. $3^{\mathrm{a}}$ ed. Artmed.

Brock, R. L., Barry, R. A., Lawrence, E., Dey, J., \& Rolffs, J. (2012). Internet Administration of Paper and Pencil Questionnaires Used in Couple Research: Assessing Psychometric Equivalence. Assessment, 19(2), 226-242. https:// doi.org/10.1177/1073191110382850

Calhoun, L. G. \& Tedeschi, R. G. (2012). Crescimento pós-traumático em intervenções clínicas cognitivo-comportamentais. Em V. E. Caballo, Manual para o Tratamento CognitivoComportamental dos Transtornos Psicológicos da Atualidade: Intervenções em crise, transtornos da personalidade e do relacionamento e psicologia da saúde (pp. 29-47). Santos.

Couto, R. N. (2019). Forças de caráter e sua relação com variáveis individuais e sociais [Tese de doutorado não publicada]. Universidade Federal da Paraíba.

Cozby, P. C. (2003). Métodos de Pesquisa em Ciências do Comportamento. Atlas.

Duarte, A. M. P. (2012). Experiência do Luto e Crescimento PósTraumático à Luz da Perspectiva da Vinculação numa Amostra de Estudantes Universitários [Dissertação de mestrado, Universidade Católica Portuguesa]. Veritati - Repositório Institucional da Universidade Católica Portuguesa. http://hdl. handle.net/10400.14/8830

Estramiana, J. L. A., Pereira, C., Monter, M. R., \& Zlobina, A. (2013). Valores Sociais. Em L. Camino, A. R. Torres, M. E. Lima, \& M. E. Pereira. (Orgs.). Psicologia social: Temas e teorias (pp. 309-344). 2nd ed. Technopolitik.
Feeney, B. C., \& Monin, J. K. (2008). An Attachment-Theoretical Perspective on Divorce. Em J. Cassidy \& P. R. Shaver (Eds.), Handbook of Attachment: Theory, Research, and Clinical Applications (pp. 934-957). 2nd ed. Guilford.

Figueiredo, L. \& Figueiredo, R. (2014). Direito Civil: Família E Sucessões. JusPODIVM.

Fonseca, C. C. (2011). Crescimento Pós-Traumático: O impacto de falar sobre os aspectos positivos de uma experiência traumática [Dissertação de mestrado, Universidade de Lisboa]. Repositório da Universidade de Lisboa. http://hdl.handle. net/10451/4937

Fonsêca, P. N., Lopes, B. J., Gusmão, E. E. S., Pessoa, V. S. A., Couto, R. N., \& Silva, M. I. F. (2017). Perdão Conjugal: Uma Explicação a Partir dos Valores Humanos. Temas em Psicologia, 25(4), 1913-1926. http://dx.doi.org/10.9788/ TP2017.4-20Pt.

Godoy, P. B. G., \& Oliveira-Monteiro, N. R. (2015). Estudo sobre Valores em Adolescentes. Psico, 46(3), 400-408. http://dx.doi. org/10.15448/1980-8623.2015.3.19426

Gouveia, V. V. (1998). La Naturaleza de los Valores Descriptores del Individualismo e del Colectivismo: Una Comparación Intra e Intercultural [Tese de doutorado não publicada] Universidade Complutense de Madri.

Gouveia, V. V. (2003). A Natureza Motivacional dos Valores Humanos: Evidências Acerca de uma Nova Tipologia. Estudos de Psicologia (Natal), 8(3), 431-443. http://dx.doi.org/10.1590/ S1413-294X2003000300010

Gouveia, V.V. (2013). Teoria Funcionalista dos Valores Humanos: Fundamentos, Aplicações e Perspectivas. Casa do Psicólogo.

Gouveia, V. V. (Org.) (2016). Teoria Funcionalista dos Valores Humanos: Áreas de Estudo e Aplicações. Vetor.

Gouveia, V. V. (2019). Human Values: Contributions from a Functional Perspective. Em S. H. Koller (ed.). Psychology in Brazil (pp. 67-81). Springer. https://doi.org/10.1007/9783-030-11336-0 5

Gouveia, V. V., Milfont, T. L., \& Guerra, V. M., (2014). Functional Theory of Human Values: Testing Its Content and Structure Hypotheses. Personality and Individual Differences, 60, 41-47. https://doi.org/10.1016/j.paid.2013.12.012

Groleau, J. M., Calhoun, L. G., Cann, A., \& Tedeschi, R. G. (2013). The Role of Centrality of Events in Posttraumatic Distress and Posttraumatic Growth. Psychological Trauma: Theory, Research, Practice, and Policy, 5(5), 477-483. https://doi. org/10.1037/a0028809

Hemphill, J. F. (2003). Interpreting the Magnitudes of Correlation Coefficients. American Psychologists, 58(1), 78-80. https:// doi.org/10.1037/0003-066X.58.1.78

Holmes, T., \& Rahe, R. (1967). The Social Readjustment Rating Scale. Journal of Psychosomatic Research, 11, 213-218. https:// doi.org/10.1016/0022-3999(67)90010-4

Instituto Brasileiro de Geografia e Estatística. (2019). Estatística do Registro Civil 2018. IBGE. 
Kulik, L., \& Heine-Cohen, E. (2011). Coping Resources, Perceived Stress, and Adjustment to Divorce among Israeli Women: Assessing Effects. The Journal of Social Psychology, 151(1), 5-30. https://doi.org/10.1080/00224540903366453

Laaser, D., Putney, H. L., Bundick, M., Delmonico, D. L., \& Griffin, E. J. (2017). Posttraumatic Growth in Relationally Betrayed Women. Journal of Marital and Family Therapy, 43(3), 435447. https://doi.org/10.1111/jmft.12211.

Lamela D., Figueiredo B., Bastos A., \& Martins H. (2014). Psychometric Properties of the Portuguese Version of the Posttraumatic Growth Inventory Short Form among Divorced Adults. European Journal of Psychological Assessment, 30(1), 3-14. https://doi.org/10.1027/1015-5759/a000161

Lamela, D., Figueiredo B., Bastos A., \& Feinberg, M. (2016). Typologies of Post-Divorce Coparenting and Parental WellBeing, Parenting Quality and Children's Psychological Adjustment. Child Psychiatry Human Development, 47(5), 716-28. https://doi.org/10.1007/s10578-015-0604-5.

Lau, J. T. F., Yeung, N. C. Y., Yu, X., Zhang, J., Mak, W. W. S., Lui, W. W. S., \& Zhang, J. (2013). Psychometric Properties of the Chinese Version of the Revised Posttraumatic Growth Inventory for Children (PTGI-C-R). Asia-Pacific Journal of Public Health, 27(2), 310-320. https://doi. org/10.1177/1010539513479967

Lindstrom, C. M., Cann, A., Calhoun, L. G., \& Tedeschi, R. G. (2013). The Relationship of Core Belief Challenge, Rumination, Disclosure, and Sociocultural Elements to Posttraumatic Growth. Psychological Trauma, 5(1), 50-55 http://dx.doi.org/10.1037/a0022030

Linley, P.A., \& Joseph, S. (2004). Positive Change Following Trauma and Adversity: A Review. Journal of Traumatic Stress, 17, 1121. https://doi.org/10.1023/B:JOTS.0000014671.27856.7e.

Marques, C., Silva, A. D., \& Taveira, M. C. (2017). Valores como Preditores da Satisfação com a Vida em Jovens. Psico-USF, 22(2), 207-215. https://doi.org/10.1590/141382712017220202.

Medeiros, E. D. (2011). Teoria Funcionalista dos Valores Humanos: Testando sua Adequação Intra e Interculturalmente [Tese de doutorado, Universidade Federal da Paraíba] Repositório Institucional da UFPB. https://repositorio.ufpb.br/jspui/handle/ tede $/ 6877$

Medeiros, E. D., Couto, R. N., Fonseca, P. N., Silva, P. G. N., \& Medeiros, P. B. C. (2017). Posttraumatic Growth Inventory (PTGI): Adaptação e Validade Fatorial no Nordeste Brasileiro. Psico-USF, 22(3), 449-460. https://doi.org/10.1590/141382712017220306

Medeiros, E. D., Couto, R. N., Fonsêca, P. N., Brito. R. C. S. \& Castro, L. S. (2016). Correlatos Valorativos do Crescimento Pós-Traumático em uma Amostra Brasileira, Psicologia e Saber Social, 5(6). 112-125. https://doi.org/10.12957/psi.saber. soc.2016.21602

Menezes, A. P. L. F. (2009). A Propensão para o Perdão na Pessoa Idosa. [Dissertação de mestrado, Universidade do Porto]. https://core.ac.uk/download/pdf/143390114.pdf

Pacico, J. C. \& Bastinello, M, R. (2014). As Origens da Psicologia Positiva e os Primeiros Estudos Brasileiros. Em C. Hutz, Avaliação em psicologia positiva (pp. 13-22). Artmed.

Ramos, C., \& Leal, I., (2013). Posttraumatic Growth in the Aftermath of Trauma: A Literature Review about Related Factors and Application Contexts. Psychology, Community \& Health, 2(1), 43-54. https://doi.org/10.5964/pch.v2i1.39.

Resende, C., Sendas, S., \& Maia,A. (2008). Estudo das características psicométricas do Posttraumatic Growth Inventory- PTGI(Inventário de Crescimento Pós-Traumático) para a população portuguesa. Em A. P. Noronha, C. Machado, L. Almeida, M. Gonçalves, S. Martind, \& V. Ramalho (Eds.), Actas da XIII
Conferência Internacional de Avaliação Psicológica: Formas $e$ Contextos (pp. 1-16). Psiquilibrios Edições.

Rokeach, M. (1973). The Nature of Human Values. Free Press.

Schwartz, S. H. (2005) Valores Humanos Básicos: Seu Contexto e Estrutura Intercultural. Em A. Tamayo \& J. B. Porto (Orgs.), Valores e comportamento nas organizações (pp. 21-55). Vozes.

Soares, A. K. S. (2015). Valores Humanos no Nivel Individual e Cultural: Um Estudo Pautado na Teoria Funcionalista [Tese de doutorado não publicada]. Universidade Federal da Paraíba.

Subandi, M. A, Achmad, T., Kurniati, T., \& Febri, R. (2014). Spirituality, Gratitude, Hope, and Post-Traumatic Growth among the Survivors of the 2010 Eruption of Mount Merapi in Java, Indonesia. Australasian Journal of Disaster and Trauma Studies, 18(1), 19-26.

Taku, K., Kilmer, R. P., Cann, A., Tedeschi, R. G., \& Calhoun, L. G. (2012). Exploring Posttraumatic Growth in Japanese Youth. Psychological Trauma: Theory, Research, Practice, and Policy, 4(4), 411-419. https://doi.org/10.1037/a0024363

Tedeschi, R. G., \& Calhoun, L. G. (1996). The Posttraumatic Growth Inventory: Measuring the Positive Legacy of Trauma. Journal of Traumatic Stress, 9(3), 455-471. https://doi.org/10.1007/ BF02103658

Tedeschi R. G., Park C. L., \& Calhoun L. G. (1998). Posttraumatic Growth: Positive Changes in the Aftermath of Crisis. Erlbaum.

Tedeschi, R. G., \& Calhoun, L. G. (2004). Posttraumatic Growth: Conceptual Foundations and Empirical Evidence. Psychological Inquiry, 15(1), 1-18. https://doi.org/10.1037/ a0024363

Triplett, K., Tedeschi, R., Cann, A., Calhoun, L., \& Reeve, C. (2012). Posttraumatic Growth, Meaning in Life, and Life Satisfaction in Response to Trauma. Psychological Trauma: Theory, Research, Practice, and Policy, 4(4), 400-410. https:// doi.org/10.1037/a0024204

Thomas, C., \& Ryan, M. (2008). Women's Perception of the Divorce Experience: A Qualitative Study. Journal of Divorce and Remarriage, 49, 210-224. https://doi. org/10.1080/10502550802222394

Ullman, S. E. (2014). Correlates of Posttraumatic Growth in Adult Sexual Assault Victims. Traumatology, 20(3) 219-224. https:// doi.org/10.1037/h0099402

Vázquez, C. (2013). Positive Psychology and Its Enemies: A Reply Based on Scientific Evidence. Papeles del Psicólogo, 34(2), 3-25.

Yárnoz-Yaben, S., Garmendia, A., \& Comino, P. (2015). Looking at the Bright Side: Forgiveness and Subjective Well-Being in Divorced Spanish Parents. Journal Happines Studies, 17(5). 1905-1919. https://doi.org/10.1007/s10902-015-9677-x

Waugh, A., Kiemle, G., \& Slade, P. (2018). What Aspects of Posttraumatic Growth are Experienced by Bereaved Parents? A Systematic Review. European Journal of Psychotraumatology, 9(1506230). https://doi.org/10.1080/20008198.2018.1506230

Wu, X., Zhou, X., Liu, L. \& Chen, J. (2014). Social Support and Deliberate Rumination Mediate the Relationship between Gratitude and Post-Traumatic Growth in Adolescents at 4.5 Years After Wenchuan Earthquake. Psychological Science, 37(5), 1148-1153.

Zhang, W., Yan, T., Du, Y., \& Liu, X. (2013). Relationship between Coping, Rumination, and Posttraumatic Growth in Mothers of Children with Autism Spectrum Disorders. Research in Autism Spectrum Disorders, 7(10), 1204-1210. https://doi. org/10.1016/j.rasd.2013.07.008

Zhou, X. \& Wu, X. (2015). Longitudinal Relationships between Gratitude, Deliberate Rumination, and Posttraumatic Growth in Adolescents Following the Wenchuan Earthquake in China. Scandinavian Journal of Psychology, 56(5), 567-572. https:// doi.org/10.1111/sjop.12237 